\title{
A Cross-Sectional Study of Overtreatment and Deintensification of Antidiabetic and Antihypertensive Medications in Diabetes Mellitus: The TEMD Overtreatment Study
}

\author{
Alper Sonmez (D) - Ilker Tasci · Ibrahim Demirci • Cem Haymana • \\ Cem Barcin - Hasan Aydin · Sevki Cetinkalp · Feyza Yener Ozturk • \\ Kamile Gul · Tevfik Sabuncu • Ilhan Satman · Fahri Bayram • \\ TEMD Study Group
}

Received: December 29, 2019 / Published online: February 22, 2020

(C) The Author(s) 2020

\begin{abstract}
Introduction: Targeting better glycated hemoglobin (HbA1c) and blood pressure (BP) goals may endanger older adults with type 2 diabetes mellitus (T2DM). Overtreatment of T2DM and hypertension is a trending issue, although undertreatment is still common. We investigated the rates and predictors of overtreatment and undertreatment of glycemia and BP in older
\end{abstract}

Enhanced Digital Features To view enhanced digital features for this article go to https://doi.org/10.6084/ m9.figshare.11770980.

Electronic supplementary material The online version of this article (https://doi.org/10.1007/s13300020-00779-0) contains supplementary material, which is available to authorized users.

A. Sonmez $(\bowtie)$

Department of Endocrinology and Metabolism, Gulhane School of Medicine, University of Health

Sciences, Ankara, Turkey

e-mail: alpersonmez@yahoo.com

I. Tasci

Department of Internal Medicine, Gulhane School of Medicine, University of Health Sciences, Ankara, Turkey

I. Demirci · C. Haymana

Department of Endocrinology and Metabolism, Gulhane Training and Research Hospital, University of Health Sciences, Ankara, Turkey adults with T2DM and physicians' attitudes to deintensify or intensify treatment.

Methods: Data from older adults ( $\geq 65$ years) enrolled in a large nationwide T2DM survey in 2017 across Turkey were analyzed. Overtreatment of glycemia was defined as $\mathrm{HbA} 1 \mathrm{c}<6.5 \%$ plus the use of $\geq 2$ oral antihyperglycemics or insulin, and BP overtreatment was defined as systolic BP (SBP) $<120 \mathrm{mmHg}$ or diastolic BP (DBP) $<65 \mathrm{mmHg}$ plus the use of $\geq 2$ drugs. Undertreatment of glycemia was defined as HbA1c $>9 \%$, and BP undertreatment was defined as $\mathrm{SBP}>150 \mathrm{mmHg}$ or DBP $>90 \mathrm{mmHg}$. Deintensification or intensification rates were calculated according to treatment modification initiated by the treating physician(s).

Results: The rate of overtreatment in the glycemia group $(n=1264)$ was $9.8 \%(n=124)$ and that in the BP group $(n=1052)$ was $7.3 \%$

C. Barcin

Department of Cardiology, Gulhane School of Medicine, University of Health Sciences, Ankara, Turkey

H. Aydin

Department of Endocrinology and Metabolism, School of Medicine, Yeditepe University, Istanbul, Turkey

S. Cetinkalp

Department of Endocrinology and Metabolism, School of Medicine, Ege University, Izmir, Turkey 
$(n=77)$, whereas the rate of undertreatment was $14.2 \% \quad(n=180)$ and $15.2 \% \quad(n=160)$, respectively. In the adjusted model, use of oral secretagogues (sulfonylureas or glinides) (odds ratio [OR] 1.94, 95\% confidence interval [CI] 1.2-3.1) and follow-up at a private clinic (OR $1.81,95 \%$ CI $1.0-3.3$ ) were predictors of glycemia overtreatment. BP overtreatment was independently associated with the use insulinbased diabetes therapies (OR 1.86, 95\% CI 1.14-3.04). There was no independent association of BP undertreatment to the study confounders. The deintensification and intensification rates were 25 and $75.6 \%$, respectively, for glycemia and 10.9 and 9.2\%, respectively, for BP.

Conclusions: The results show that one in ten older adults with T2DM are overtreated while one in four require modification of their current antihyperglycemic and antihypertensive treatments. Physicians are eager to intensify medications while they largely ignore deintensification in diabetes management. These results warrant enforced measures to improve the care of older adults with T2DM.

Trial Registration: ClinicalTrials.gov identifier, NCT 03455101.

F. Y. Ozturk

Department of Endocrinology and Metabolism, Sisli

Hamidiye Etfal Training and Research Hospital,

University of Health Sciences, Istanbul, Turkey

K. Gul

Department of Endocrinology and Metabolism, School of Medicine, Sutcu Imam University,

Kahramanmaras, Turkey

T. Sabuncu

Department of Endocrinology and Metabolism,

School of Medicine, Harran University, Sanliurfa,

Turkey

I. Satman

Department of Endocrinology and Metabolism, School of Medicine, Istanbul University, Istanbul, Turkey

F. Bayram

Department of Endocrinology and Metabolism, School of Medicine, Erciyes University, Kayseri, Turkey

\section{PLAIN LANGUAGE SUMMARY}

Type 2 diabetes mellitus (T2DM) is prevalent in the elderly population. Strict glycemia and blood pressure (BP) targets do not improve outcomes but they may increase the rate of adverse events in these patients. Consequently, overtreatment has been an emerging issue in recent years. The overall magnitude of the problem is largely unknown. Therefore, we assessed the rates and predictors of overtreatment and undertreatment of blood glucose and BP in Turkish older adults with T2DM. We also investigated physicians' reactions to treatment modulation in the overtreated or undertreated patients. Patients participating in the study were: older adults $(n=1264)$ with T2DM from a cross-sectional, nationwide, multicenter study who followed-up at the same unit during the past 12 months and who did not have decompensated liver disease, psychiatric disorders interfering with cognition or compliance, bariatric surgery in the past 12 months or renal replacement therapy. We found that:

- One of ten older adults with T2DM were overtreated for glycemia.

- One in four older adults with T2DM required modification of antihyperglycemic treatment with inclusion of the number of insufficiently treated individuals.

- One in four older adults with T2DM required modification of antihypertensive treatment.

- Physicians are much more inclined to intensify antihyperglycemia medications, while they largely ignore the need for deintensification in the overtreated patients.

- Physicians did not modify antihypertensive treatments in about $90 \%$ of patients with uncontrolled BP.

- Patients who were treated by oral secretagogues and followed in private clinics were most prone to glycemia overtreatment.

- Patients who were treated by insulin-based regiments were prone to BP overtreatment. 
These results suggest that measures should be taken to improve physician awareness of drug modification in older patients with T2DM.

Keywords: Arterial blood pressure; Glycemia; Older adults; Overtreatment; Type 2 diabetes; Undertreatment

\section{Key Summary Points}

\section{Why carry out this study?}

Overtreatment of glycemia and blood pressure (BP) is a critical issue but one that is often overlooked in older adults with type 2 diabetes mellitus (T2DM).

We determined the frequency of overtreatment and undertreatment of glycemia and blood pressure (BP) in a nationwide population of older adults.

The attitudes of physicians to treatment modulation in these patients were also investigated.

\section{What was learned from the study?}

One in every four older adults with T2DM needs treatment modulation for BP or glycemia regulation.

Although not more frequent than undertreatment, overtreatment of glycemia is a common occurrence, noted in one in every ten older adults.

Physicians are eager to intensify medications while they largely ignore deintensification in diabetes management.

It is critical to target the optimal, not lower, levels of glycemic and BP in the management of older adults with T2DM.

\section{INTRODUCTION}

Diabetes mellitus is one of the most common chronic diseases worldwide, leading to serious public health problems and a significant economic burden $[1,2]$. The prevalence of diabetes mellitus is higher among the elderly population and is expected to further increase in the future due to the increasing life expectancy [3]. The framework of diabetes management is well drawn in adults, yet the situation in elderly patients is somewhat different [4]. Older patients who have multiple comorbidities, limited life expectancy, and increased risk of hypoglycemia may derive less benefits from stringent glycemic control and lower blood pressure (BP) goals [5]. In these patients, the risk of adverse events likely exceeds some of the benefits of standard care that are achieved in younger individuals with diabetes.

Landmark studies have shown that tight glycemic and BP control are not only ineffective in improving outcomes but also associated with more adverse events in older patients with type 2 diabetes mellitus (T2DM) [6-9]. Thus, the most recent guidelines have included specific sections for the management of T2DM in older adults in which looser glycated hemoglobin (HbA1c) goals are recommended. The American Diabetes Association (ADA), European Association for the Study of Diabetes (EASD), and the Turkish Society of Endocrinology and Metabolism (TEMD) have all recommended personalized $\mathrm{HbA1c}$ targets of between 6.5 and $8.5 \%$ according to age, additional comorbidities, and life expectancy [10-12]. The American Geriatrics Society (AGS) has also indicated that medications other than metformin should be avoided when an older patient's HbA1c is $<6.5 \%$ [13], primarily due to the decreased potential of benefits and increased risk of morbidity and mortality related to the side effects of such medications [13-15]. Similar to its recommendations for glycemia management, the ADA has established personalized BP targets in patients with diabetes mellitus that recommend lower goals in older individuals [16]. The Turkish Hypertension Consensus Report defines the BP targets in older adults with diabetes mellitus as 130-140/70-80 $\mathrm{mmHg}$ [17]. 
Following these updates, overtreatment of T2DM has become an emerging issue, leading to the publication of new findings from several countries. Although the proportion of patients with excessively low HbA1c or BP level does not appear to be critical, such overtreated patients are multimorbid, suggesting that the problem may have serious consequences for the health status of older adults. As in many other countries, the magnitude of the problem in Turkish patients is largely unknown [18]. Therefore, our aim in this study was to assess the rates and predictors of overtreatment and undertreatment of blood glucose and BP in older adults with T2DM. We also investigated physicians' reactions to treatment modulation in the overtreated or undertreated patients.

\section{METHODS}

\section{Study Design}

Patients aged $\geq 65$ years from the Turkish Nationwide Survey of Glycemic and Other Metabolic Parameters of Patients with Diabetes Mellitus (TEMD) study [19] were enrolled in the present study. The TEMD study was a crosssectional, nationwide, multicenter survey involving 68 tertiary endocrine units from 37 cities throughout Turkey. Data were collected between 1 April and 30 June 2017, and the patients were allocated according to the 12 NUTs (Nomenclature of Territorial Units for Statistics regions; EU designation) of the country.

The study was conducted in accordance with the Helsinki Declaration of 1964, as revised in 2013. The local (University of Health Sciences, Kecioren Training and Research Hospital Ethical Committee: no. 131309) and central (Republic of Turkey, Ministry of Health, Pharmaceuticals and Medical Devices Agency: no. 14-MAR-2017/93189304-514.11.01-E.58933) ethics committees approved the study protocol, and participants provided informed consent to participate. This study is registered at ClinicalTrials.gov (NCT 03455101).

\section{Study Population}

Outpatients with a previous diagnosis of T2DM who were having a regular control evaluation were enrolled in the study. The study protocol required that all participants had been followed-up at the same unit during the past 12 months. Exclusion criteria were decompensated liver disease, psychiatric disorders interfering with cognition or compliance, bariatric surgery in the past 12 months, and having received/receiving renal replacement therapy.

\section{Data Collection}

Data were collected from physicians using a questionnaire, which was administered in faceto-face interviews. The questionnaire included demographic information, length of diabetes mellitus, smoking, number of HbA1c measurements annually, type of diabetes care center (state hospital or private), personal diabetes management (diet and exercise), medications, macrovascular complications, microvascular complications, comorbid diseases, and the frequency of hypoglycemic episodes.

Height and weight of the patients were measured with the patients in their underwear according to a standard protocol. Body mass index (BMI) was computed as the ratio of weight to the square of height $\left(\mathrm{kg} / \mathrm{m}^{2}\right)$. Arterial $\mathrm{BP}$ was measured using an automatic BP monitor (Omron M2 HEM-7121-E; Omron Corp., Kyoto, Japan) with the patient in the sitting position after at least 5 min of rest; three consecutive measurements were made on the same arm, and the mean was recorded. Home BP was measured twice daily for 1 week, and the mean of these measurements was recorded at the control visit.

Participating physicians recorded the laboratory test results at their sites. Blood samples were drawn from the antecubital vein, between 0800 and 1000 hours, after overnight fasting. The fasting blood concentration of glucose, total and high-density lipoprotein cholesterol level, and triglycerides level were measured using an enzymatic method. Low-density cholesterol was calculated using the 
Friedewald's equation if the triglyceride level was $<400 \mathrm{mg} / \mathrm{dL}$ [20]. HbA1c was measured using high-performance liquid chromatography, turbidimetric inhibition immunoassay, or enzymatic methods.

\section{Definitions of Study Variables}

Overtreatment for glycemia was defined as HbA1c $<6.5 \%$ plus the use of $\geq 2$ oral antihyperglycemics or insulin [21]. Undertreatment for glycemia was defined as HbA1c $>9 \%$. Overtreatment for BP was defined as systolic BP (SBP) $<120 \mathrm{mmHg}$ or diastolic BP (DBP) $<65 \mathrm{mmHg}$ when a patient was receiving $\geq 2$ antihypertensive drugs. Undertreatment for $\mathrm{BP}$ was defined as SBP $>150 \mathrm{mmHg}$ or DPB $>90 \mathrm{mmHg}$ [22]. In our analysis, we used home BP readings when they were available, otherwise the office readings were used for analysis. Deintensification was defined as the discontinuation or dose reduction of drugs by the treating physician according to BP readings and $\mathrm{HbA} 1 \mathrm{c}$ results.

A positive history of physical exercise was defined as exercising/participating in physical activities for $>30$ min at least 3 days per week. Hypoglycemia was defined according to patient reports of typical adrenergic symptoms of hypoglycemia, with a concomitant capillary glucose level of $\leq 70 \mathrm{mg} / \mathrm{dL}$. Participating sites were coded as a private care facility if they were not publicly governed. Lower education indicates an attained level of education of $<8$ years. Macrovascular complications were either selfreported, as having a history of coronary heart disease, angina, heart attack, cerebrovascular event or peripheral artery disease; or recorded by the physicians according to their findings, such as non-palpable lower extremity pulses, lower ankle-brachial index values $(\leq 0.9)$, positive findings on coronary or peripheral arteriography, or positive results in carotid or peripheral arterial duplex ultrasound examination. Retinopathy was self-reported based on the results of routine eye examinations in the medical history. Nephropathy was recorded as positive if the patient had albuminuria and/or decreased estimated glomular filtration rate, and was either self-reported or recorded by the physicians based on the symptoms of typical bilateral symmetric distal neuropathy or other autonomous neuropathies attributable to diabetes mellitus.

\section{Statistical Analyses}

Statistical analysis was performed in SPSS 18.0 (IBM Corp., Armonk, NY, USA). Data were expressed as the mean \pm standard deviation for continuous variables or as the percentage for categorical variables. Comparisons between groups were performed using the one-way analysis of variance test for continuous variables. The Chi-square test was used for categorical comparisons. Binominal logistic regression was performed to ascertain the effects of different variables on the likelihood of overtreatment or undertreatment status. Significance was set at 0.05 (two-tailed $p$ value of $\leq 0.05)$.

\section{RESULTS}

The TEMD study included 5211 patients aged $\geq$ 18 years. After excluding patients with type 1 diabetes mellitus $(n=455)$ and younger individuals $(n=3492), 1264$ older patients were eligible for the evaluation of glycemic targets. Within this group, 1052 patients were eligible for the evaluation of BP targets.

\section{Diabetes Overtreatment, Optimal Treatment, and Undertreatment}

The final sample of 1264 older patients included in this study for evaluation of their glycemic target included 124 (9.8\%) who were overtreated, 960 (76\%) who were optimally treated, and $180(14.2 \%)$ who were undertreated. Table 1 shows the demographic and metabolic characteristic of patients based on glycemic control. Mean age, gender, smoking, and the ratio of hypoglycemic events were not different in the overtreated, optimally treated, or undertreated patients. In general, overtreated patients had a higher education level, a shorter duration 
Table 1 Demographic and metabolic parameters of older patients with type 2 diabetes mellitus based on glycemic control

\begin{tabular}{|c|c|c|c|c|}
\hline $\begin{array}{l}\text { Demographic and metabolic } \\
\text { parameters }\end{array}$ & $\begin{array}{l}\text { Overtreated } \\
(n=124 ; 9.8 \%)^{a}\end{array}$ & $\begin{array}{l}\text { Optimally treated } \\
(n=960 ; 75.9 \%)\end{array}$ & $\begin{array}{l}\text { Undertreated } \\
(n=180 ; 14.2 \%)^{\mathrm{a}}\end{array}$ & $p$ \\
\hline Age (year) & $70.2 \pm 4.7$ & $71.2 \pm 5.3$ & $70.5 \pm 5.2$ & 0.056 \\
\hline \multicolumn{5}{|l|}{ Gender } \\
\hline Female & $68(54.8 \%)$ & $545(56.1 \%)$ & $111(61.3 \%)$ & \multirow[t]{2}{*}{0.390} \\
\hline Male & $57(45.2 \%)$ & $425(43.9 \%)$ & $70(38.7 \%)$ & \\
\hline Diabetes duration (year) & $13.0 \pm 7.5$ & $14.2 \pm 9.1$ & $16.7 \pm 8.7$ & 0.001 \\
\hline BMI $\left(\mathrm{kg} / \mathrm{m}^{2}\right)$ & $30.5 \pm 6.5$ & $31.1 \pm 5.7$ & $32.1 \pm 6.8$ & 0.048 \\
\hline HbAlc (\%) & $6.08 \pm 0.33$ & $7.23 \pm 0.89$ & $10.47 \pm 1.58$ & $<0.001$ \\
\hline $\begin{array}{l}\text { Exercise }(>2 \text { days/week } \\
\text { and }>90 \mathrm{~min} / \text { week })\end{array}$ & $25(20.5 \%)$ & $162(17.2 \%)$ & $18(10.0 \%)$ & 0.027 \\
\hline Smoking & $4(3.2 \%)$ & $58(6.1 \%)$ & $11(6.1 \%)$ & 0.435 \\
\hline \multicolumn{5}{|l|}{ HbAlc measurement (\%; annually) } \\
\hline $0-2$ & $52(46.0 \%)$ & $377(42.9 \%)$ & $99(62.3 \%)$ & \multirow[t]{3}{*}{$<0.001$} \\
\hline $3-4$ & $56(49.6 \%)$ & $463(52.7 \%)$ & $55(34.6 \%)$ & \\
\hline$\geq 5$ & $5(4.4 \%)$ & $38(4.3 \%)$ & $5(3.1 \%)$ & \\
\hline \multicolumn{5}{|l|}{ Blood pressure } \\
\hline SBP & $131.6 \pm 15.6$ & $135.7 \pm 19.2$ & $137.1 \pm 19.6$ & \multirow[t]{2}{*}{0.037} \\
\hline DBP & $77.9 \pm 9.8$ & $79.0 \pm 10.9$ & $79.3 \pm 11.3$ & \\
\hline \multicolumn{5}{|l|}{ Type of medical center } \\
\hline Private care center & $20(16.1 \%)$ & $102(10.5 \%)$ & $11(6.1 \%)$ & \multirow[t]{2}{*}{0.018} \\
\hline State hospital & $104(83.9 \%)$ & $869(89.5 \%)$ & $170(93.9 \%)$ & \\
\hline \multicolumn{5}{|l|}{ Education } \\
\hline $\begin{array}{l}\text { Higher education (high school or } \\
\text { university) }\end{array}$ & $51(41.8 \%)$ & $351(36.5 \%)$ & $49(27.8 \%)$ & \multirow[t]{2}{*}{0.031} \\
\hline $\begin{array}{l}\text { Lower education (illiterate, primary } \\
\text { and secondary school) }\end{array}$ & $71(58.2 \%)$ & $611(63.5 \%)$ & $127(72.2 \%)$ & \\
\hline \multicolumn{5}{|l|}{ Complications } \\
\hline Macrovascular & $38(30.6 \%)$ & $342(35.2 \%)$ & $85(47.0 \%)$ & 0.004 \\
\hline Microvascular & $57(46.0 \%)$ & $504(51.9 \%)$ & $124(68.5 \%)$ & $<0.001$ \\
\hline Hypoglycemic event & $53(43.1 \%)$ & $407(42.6 \%)$ & $79(44.4 \%)$ & 0.904 \\
\hline \multicolumn{5}{|l|}{ Treatment } \\
\hline Insulin based & $43(34.7 \%)$ & $483(49.7 \%)$ & $118(65.2 \%)$ & \multirow[t]{2}{*}{$<0.001$} \\
\hline Non-insulin based & $81(65.3 \%)$ & $488(50.3 \%)$ & $63(34.8 \%)$ & \\
\hline
\end{tabular}


Table 1 continued

\begin{tabular}{lllll}
\hline $\begin{array}{l}\text { Demographic and metabolic } \\
\text { parameters }\end{array}$ & $\begin{array}{l}\text { Overtreated } \\
(\boldsymbol{n}=\mathbf{1 2 4} ; \mathbf{9 . 8 \%})^{\mathbf{a}}\end{array}$ & $\begin{array}{l}\text { Optimally treated } \\
(\boldsymbol{n}=\mathbf{9 6 0} ; \mathbf{7 5 . 9 \% )})\end{array}$ & $\begin{array}{l}\text { Undertreated } \\
(\boldsymbol{n}=\mathbf{1 8 0} ; \mathbf{1 4 . 2 \%})^{\mathbf{a}}\end{array}$ & $\boldsymbol{p}$ \\
\hline OAD (0-2 drug) & $102(82.3 \%)$ & $845(87.0 \%)$ & $164(90.6 \%)$ & 0.102 \\
OAD ( $\geq 3$ drugs) & $22(17.7 \%)$ & $126(13.0 \%)$ & $17(9.4 \%)$ & \\
Chronic conditions & & & $20(11.0 \%)$ & $<\mathbf{0 . 0 0 1}$ \\
$0-1$ & $22(17.7 \%)$ & $119(12.3 \%)$ & $90(49.7 \%)$ & \\
$2-3$ & $78(62.9 \%)$ & $604(62.2 \%)$ & $71(39.2 \%)$ & \\
$4+$ & $24(19.4 \%)$ & $248(25.5 \%)$ & \\
\hline
\end{tabular}

Data in table are expressed as the mean \pm standard deviation (SD) (continuous variables) or as the number with the percentage in parenthesis (categorical variables)

$p$-values are derived from ANOVA and Chi-square tests for continuous and categorical variables, respectively

$p$ values $<0.05$ are given in bold

$B M I$ Body mass index, $D B P$ diastolic blood pressure, $H b A 1 c$ glycated hemoglobin, $O A D$ oral antidiabetic drug, $S B P$ systolic blood pressure

${ }^{a}$ Overtreatment for glycemia was defined as HbAlc of $<6.5 \%$ plus the use of $\geq 2$ oral antihyperglycemics or insulin ; undertreatment for glycemia was defined as HbAlc $>9 \%$.

of diabetes, lower BMI, higher physical activity, more frequent HbA1c measurements, and lower mean BP level. Overtreated individuals were found to be more frequently under private care follow-up and, overall, $10.5 \%$ of overtreated patients $(n=133)$ were recruited from private care centers (Table 1). Overtreated patients also had lower rates of macrovascular and microvascular complications, fewer insulinbased treatments, fewer comorbidities, and a higher number of antihyperglycemics than their optimally treated or undertreated counterparts. Of the patients using antihyperglycemics in the overtreatment, optimal treatment and undertreatment groups, metformin was used by $96.0,72.7$, and $65.6 \%$, respectively $(p<0.001)$; secretagogues by 47.6 , 27.3 , and $27.2 \%$, respectively $(p<0.001)$, dipeptidyl peptidase-4 inhibitors by $42.7,28.9$, and $25.6 \%$, respectively $(p=0.003)$; pioglitazone by $6.5,2.1$, and $2.8 \%$, respectively $(p=0.016)$; acarbose by $5.6,3.9$, and $2.2 \%$, respectively ( $p=0.303)$, sodium-glucose transport protein 2 (SGLT2) inhibitors by $0.8,1.3$, and $1.1 \%$, respectively $(p=0.907)$, and injectable treatments with/without oral drugs by $65,49.2$, and $34.7 \%$, respectively $(p<0.001)$.

\section{Blood Pressure Overtreatment, Optimal Treatment, and Undertreatment}

The final sample of 1052 older patients with hypertension included in this study for evaluation of their BP target included 77 (7.3\%) who were overtreated, $815(77.5 \%)$ who were optimally treated, and 160 (15.2\%) who were undertreated. Table 2 shows the demographic and metabolic characteristics of this patient sample based on BP treatment. Mean age, gender, BMI, diabetes duration, HbA1c, ratio of higher education, weekly exercise frequency, smoking, macrovascular complications, ratio of hypoglycemic events, number of oral antihyperglycemics, and number of chronic conditions were not different in overtreated, optimally treated or undertreated patients. Overall, $11.2 \%$ of patients $(n=118)$ were recruited from private care centers. There was no difference between the private and state hospitals in terms of number of overtreated, undertreated or optimal treated patients (Table 2). There were significant differences in the rates of microvascular complications, insulin-based treatments, and the number of antihypertensive drugs across the three patient groups. 
Table 2 Demographic and metabolic parameters of older patients with type 2 diabetes mellitus based on blood pressure control

\begin{tabular}{|c|c|c|c|c|}
\hline $\begin{array}{l}\text { Demographic and metabolic } \\
\text { parameters }\end{array}$ & $\begin{array}{l}\text { Overtreated } \\
(n=77 ; 7.3 \%)^{a}\end{array}$ & $\begin{array}{l}\text { Optimally treated } \\
(n=815 ; 77.5 \%)\end{array}$ & $\begin{array}{l}\text { Undertreated } \\
(n=160 ; 15.2 \%)^{a}\end{array}$ & $p$ \\
\hline Age (year) & $71.7 \pm 5.0$ & $71.2 \pm 5.3$ & $71.1 \pm 5.0$ & 0.704 \\
\hline \multicolumn{5}{|l|}{ Gender } \\
\hline Female & $45(58.4 \%)$ & $473(58.0 \%)$ & $94(58.8 \%)$ & \multirow[t]{2}{*}{0.985} \\
\hline Male & $32(41.6 \%)$ & $342(42.0 \%)$ & $66(41.3 \%)$ & \\
\hline BMI $\left(\mathrm{kg} / \mathrm{m}^{2}\right)$ & $31.3 \pm 6.6$ & $31.6 \pm 5.8$ & $32.0 \pm 6.1$ & 0.611 \\
\hline Diabetes duration (year) & $16.0 \pm 8.4$ & $14.5 \pm 9.0$ & $14.8 \pm 7.9$ & 0.359 \\
\hline HbAlc (\%) & $7.48 \pm 1.40$ & $7.61 \pm 1.57$ & $7.60 \pm 1.58$ & 0.797 \\
\hline \multicolumn{5}{|l|}{ Blood pressure } \\
\hline SBP & $113.6 \pm 12.1$ & $134.5 \pm 15.1$ & $160.8 \pm 16.1$ & $<0.001$ \\
\hline DBP & $65.5 \pm 8.4$ & $79.1 \pm 9.6$ & $89.6 \pm 10.1$ & $<0.001$ \\
\hline \multicolumn{5}{|l|}{ Types of medical center } \\
\hline Private care center & $13(16.9 \%)$ & $92(11.3 \%)$ & $13(8.1 \%)$ & \multirow[t]{2}{*}{0.134} \\
\hline State hospital & $64(83.1 \%)$ & $723(88.7 \%)$ & $147(91.9 \%)$ & \\
\hline \multicolumn{5}{|l|}{ Education } \\
\hline $\begin{array}{l}\text { Higher education (high school or } \\
\text { university) }\end{array}$ & $21(27.6 \%)$ & $284(35.3 \%)$ & $60(38.0 \%)$ & \multirow[t]{2}{*}{0.294} \\
\hline $\begin{array}{l}\text { Lower education (illiterate, primary } \\
\text { and secondary school) }\end{array}$ & $55(72.4 \%)$ & $520(64.7 \%)$ & $98(62.0 \%)$ & \\
\hline $\begin{array}{r}\text { Exercise }(>2 \text { day/week } \\
\text { and }>90 \mathrm{~min} / \text { week })\end{array}$ & $10(13.3 \%)$ & $135(16.9 \%)$ & $23(14.4 \%)$ & 0.578 \\
\hline Smoking & $4(5.2 \%)$ & $41(5.0 \%)$ & $7(4.4 \%)$ & 0.934 \\
\hline \multicolumn{5}{|l|}{ Complications } \\
\hline Macrovascular complications & $37(48.1 \%)$ & $321(39.4 \%)$ & $63(39.4 \%)$ & 0.327 \\
\hline Microvascular complications & $49(63.6 \%)$ & $434(53.3 \%)$ & $105(65.6 \%)$ & 0.006 \\
\hline Hypoglycemic event & $41(53.2 \%)$ & $337(41.9 \%)$ & $72(45.3 \%)$ & 0.136 \\
\hline \multicolumn{5}{|l|}{ Treatment } \\
\hline Insulin based & $51(66.2 \%)$ & $405(49.7 \%)$ & $90(56.3 \%)$ & \multirow[t]{2}{*}{0.010} \\
\hline Non-insulin based & $26(33.8 \%)$ & $410(50.3 \%)$ & $70(43.8 \%)$ & \\
\hline OAD (0-2 drugs) & $70(90.9 \%)$ & $694(85.2 \%)$ & $145(90.6 \%)$ & \multirow[t]{2}{*}{0.089} \\
\hline $\mathrm{OAD}(\geq 3$ drugs $)$ & $7(9.1 \%)$ & $121(14.8 \%)$ & $15(9.4 \%)$ & \\
\hline \multicolumn{5}{|l|}{ Anti-hypertensive drug } \\
\hline$\geq 3$ drugs & $59(76.7 \%)$ & $146(17.9 \%)$ & $33(20.6 \%)$ & $<0.001$ \\
\hline
\end{tabular}


Table 2 continued

\begin{tabular}{lllll}
\hline $\begin{array}{l}\text { Demographic and metabolic } \\
\text { parameters }\end{array}$ & $\begin{array}{l}\text { Overtreated } \\
(\boldsymbol{n}=\mathbf{7 7 ; 7 . 3 \%})^{\mathbf{a}}\end{array}$ & $\begin{array}{l}\text { Optimally treated } \\
(\boldsymbol{n}=\mathbf{8 1 5} ; \mathbf{7 7 . 5 \%})\end{array}$ & $\begin{array}{l}\text { Undertreated } \\
(\boldsymbol{n}=\mathbf{1 6 0} \mathbf{1 5 . 2 \%})^{\mathbf{a}}\end{array}$ & $\boldsymbol{p}$ \\
\hline Chronic conditions & & & & \\
$0-1$ & $4(5.2 \%)$ & $41(5.0 \%)$ & $8(5.0 \%)$ & 0.404 \\
$2-3$ & $41(53.2 \%)$ & $518(63.6 \%)$ & $105(65.6 \%)$ & \\
$4+$ & $32(41.6 \%)$ & $256(31.4 \%)$ & $47(29.4 \%)$ & \\
\hline
\end{tabular}

Data in table are expressed as the mean \pm SD (continuous variables) or as the number with the percentage in parenthesis (categorical variables)

$p$ values are derived from ANOVA and Chi-square tests for continuous and categorical variables, respectively $p$ values $<0.05$ are given in bold

$B M I$ Body mass index, $D B P$ diastolic blood pressure, $H b A 1 c$ glycated hemoglobin, $O A D$ oral antidiabetic drug, $S B P$ systolic blood pressure

a Overtreatment for $\mathrm{BP}$ was defined as $\mathrm{SBP}<120 \mathrm{mmHg}$ or $\mathrm{DBP}<65 \mathrm{mmHg}$ when a patient was receiving $\geq 2$ antihypertensive drugs. Undertreatment for BP was defined as SBP $>150 \mathrm{mmHg}$ or DPB $>90 \mathrm{mmHg}$

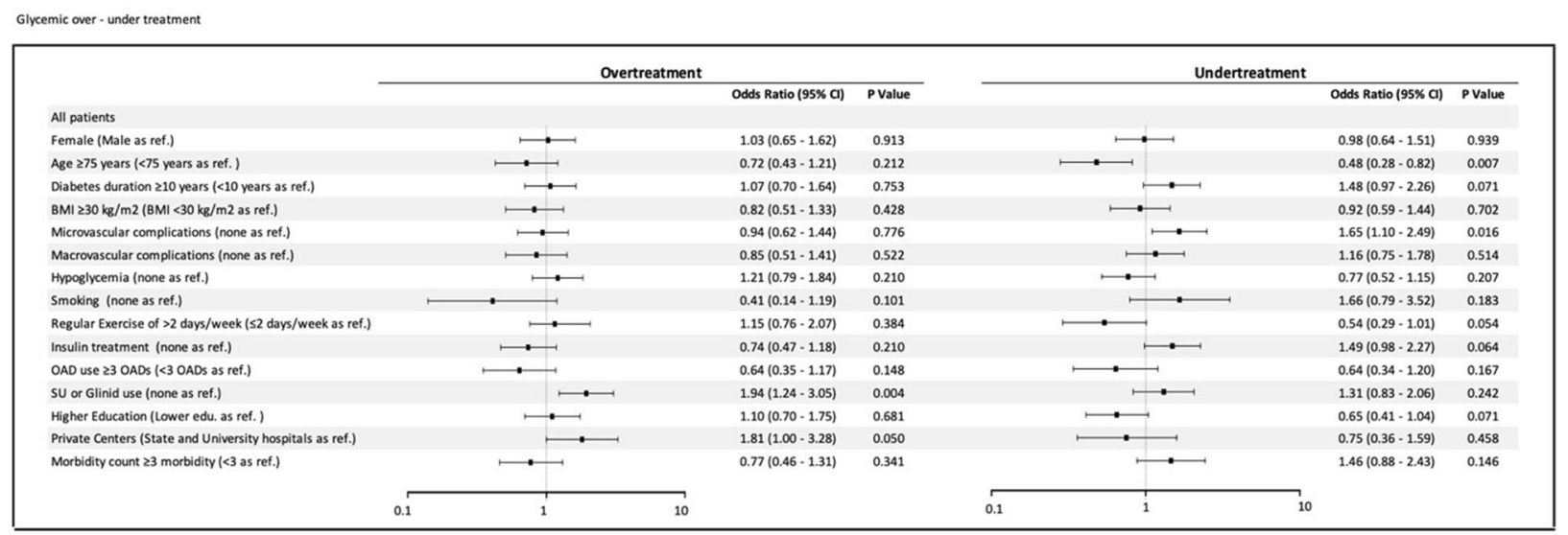

Fig. 1 Patient characteristics independently associated with glycemia overtreatment and undertreatment, respectively. $B M I$ Body mass index, $C I$ confidence interval, $O A D$ oral antidiabetic drug, $S U$ sulfonylureas

According to the multivariate analyses, the use of oral secretagogues (sulfonylureas or glinides) (odds ratio [OR] 1.94, 95\% confidence interval [CI] 1.2-3.1) and follow-up at a private clinic (OR 1.81, 95\% CI 1.0-3.3) were independent predictors of glycemia overtreatment. Age $>75$ years (OR $0.48,95 \%$ CI $0.28-0.82$ ) and the presence of microvascular complications (OR 1.63, 95\% CI 1.1-2.5) were independently associated with glycemia undertreatment (Fig. 1).

Blood pressure overtreatment was independently associated with the use of insulin-based diabetes treatment (OR 1.86, 95\% CI 1.14-3.04).
There was no independent association of BP undertreatment to the study confounders (Fig. 2).

\section{Modification of Treatment in Overtreated and Undertreated Patients}

Participating physicians deintensified antihyperglycemic treatment in $25 \%$ of subjects who were overtreated for glycemia and intensified treatment in $75.6 \%$ of subjects who were above the recommended HbA1c goal.

Deintensification of antihypertensives was made in $10.9 \%$ of patients with low BP, and intensification was made in $9.2 \%$ of subjects with uncontrolled BP. 


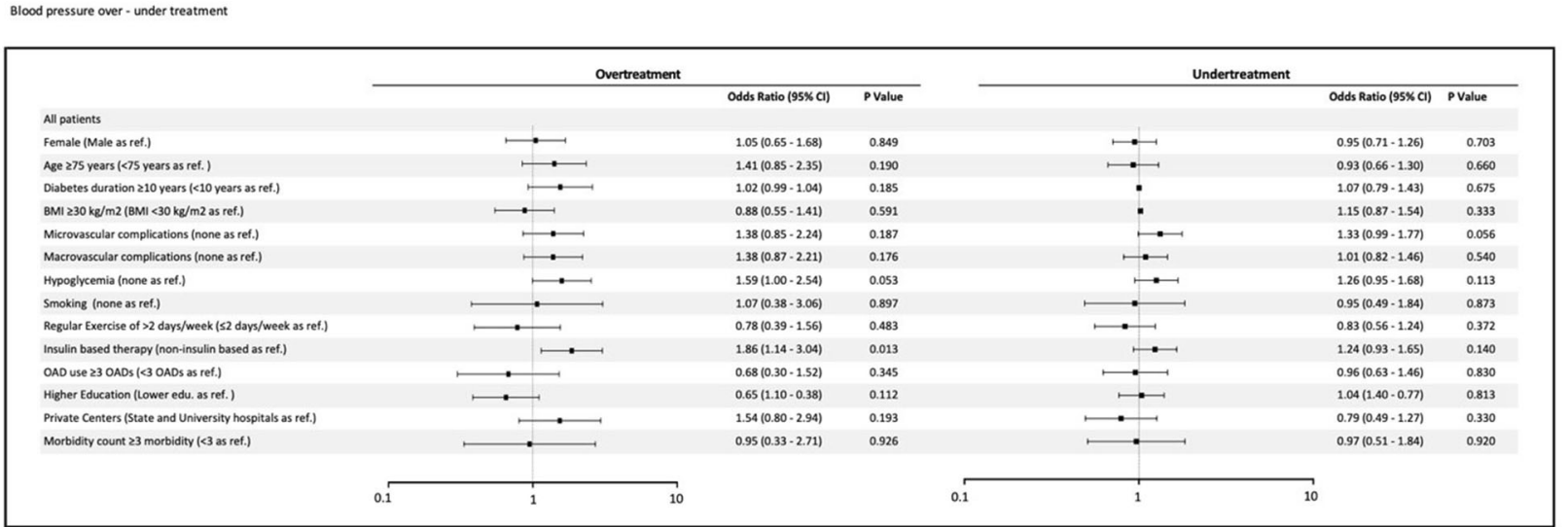

Fig. 2 Patient characteristics independently associated with blood pressure overtreatment and undertreatment, respectively

\section{DISCUSSION}

Analysis of the patient population of this study revealed that one of ten older adults with T2DM were overtreated for glycemia. When the number of insufficiently treated individuals were included in the analysis, one in four older adults with T2DM undergoing a routine control evaluation required modification of their antihyperglycemic treatment. Almost a similar proportion required modification of their antihypertensive treatment, but overtreatment of BP was overall less common. Our findings also show that participating physicians deintensified treatment in only $25 \%$ of patients overtreated for glycemia and in only $10.9 \%$ of patients overtreated for BP. In comparison, physicians intensified diabetes treatment in the majority of those with uncontrolled glycemia but did not modify the antihypertensive treatment in the $>$ $90 \%$ of patients with uncontrolled BP.

Both the ADA and AGS recommend more liberal HbA1c goals for older patients, particularly in those with functional limitations $[10,15]$ as functional decline may be considered as one of the major complications of diabetes mellitus over the long term. In a sample of patients from the Atherosclerosis Risk in Communities Study, diabetes mellitus was associated with functional disability in older adults that could not be explained by known risk factors and comorbidities, including poor glycemic control and medication use [23]. Thus, these patients may benefit from regular assessment of functionality even though the level of evidence to support this recommendation is low [10].

Studies published to date have shown a variable frequency of overtreatment of diabetes mellitus among older adults. A recent analysis of data extracted from a large US Medicare claims database revealed that $10.8 \%$ of patients aged $\geq 65$ years were potentially overtreated [21]. Overtreatment in that study was defined as $\mathrm{HbA} 1 \mathrm{c}<6.5 \%$ plus the use of any antihyperglycemic drug in addition to metformin. In the current survey, we selected a more stringent criterion for diabetes overtreatment (HbA1c < $6.5 \%$ plus the use of $\geq 2$ oral antihyperglycemic drugs or any insulin) but found that the proportion of overtreated individuals was similar to that in the US sample. However, it is likely that both studies underestimated the number of overtreated patients because HbA1c goals are currently not less than 7\% for all older adults, and higher thresholds are defined for individuals with multimorbidity and/or functional dependence $[10,13]$. Nevertheless, in the absence of evidence from randomized controlled trials (RCTs), there is no agreement on the definition of overtreatment, intensive treatment, or excessive treatment [24], and these terms have been used interchangeably.

Low adherence to treatment is a known challenge in diabetes management [25-27]. However, overemphasizing metabolic control may cause excessive treatment of persons with T2DM of all ages. In the present study, overtreated patients had better sociodemographic 
characteristics and more favorable care indicators than those who were optimally treated or undertreated. In addition, overtreated patients more often chose for follow-up in a private clinic and were more often on oral antihyperglycemics than injectable drugs. These findings suggest that medical treatment unexpectedly went too far regarding the management of the overtreated patients in our sample. In a US sample of patients with T2DM, Maciejewski et al. reported that individuals enrolled in a Medicaid program were potentially overtreated compared to those not enrolled in a Medicaid program [21], suggesting that consistent provision of diabetes care improves health-related outcomes. Since all of our participants were covered by the state health insurance, we were not able to examine the interaction between the health insurance program and overtreatment. However, follow-up at a private clinic was a predictor of overtreatment of T2DM in our study. Although what makes a patient choose a private setting can be multifactorial [28], a private clinic may offer more personal care within sufficient time and may provide a more convenient environment compared to public facilities. Also, a private clinic setting can improve physician-patient agreement, which is a predictor of medication compliance in the primary care setting [29]. Moreover, favorable health beliefs and provision of better illness-related support are positively associated with adherence to health-promoting activities consisting of a diabetic regimen and metabolic control $[30,31]$. In a RCT, Munshi and colleagues showed that the assessment and resolution of barriers to self-care improved glycemia and quality of care among older adults over a period of 12 months [32]. Nevertheless, the results of our study suggest that sustained glycemic control through management at private clinics may unnecessarily result in excessive $\mathrm{HbA1c}$ reductions over time. Increasing the awareness of overtreatment among both physicians and patients may balance personal expectations and evidence-based care requirements in the management of T2DM.

In the present study, overtreatment of patients with diabetes mellitus was independently associated with the use of secretagogues, but treatment with insulin did not show any association with overtreatment. In two large studies from the USA, overtreated patients with diabetes mellitus were more frequently on insulin and sulfonylureas [33], and the proportion of the users of both drugs did not decrease in advanced age groups [34]. Sulfonylureas are the second most prescribed antihyperglycemics across Europe [35], but they are also potent antihyperglycemics and associated with a wellknown risk of hypoglycemia [36]. Our findings suggest that sulfonylureas may cause excessive HbA1c reductions over time in older adults with T2DM; however, they are certainly agents that are less associated with the risk of hypoglycemia and even reduce the risk of cardiovascular events. Thus, when the glycemia is near normal and there are no apparent symptoms, cessation of treatment with an otherwise beneficial drug may also be arguable. It should be noted that in our study the use of such agents (e.g., SGLT-2 inhibitors, glucagon-like peptide 1 analogues) were not more common in the overtreatment group than in the other two groups.

Management of hypertension has long been complicated by concerns related to the excessive treatment of high BP [37]. A cornerstone finding in this regard was the report of a relation of lower DBP to increased risk of major cardiovascular events in the general population, which was more pronounced in the presence of diabetes mellitus [38]. Currently, based on evidence from RCTs [6], SBP $<120 \mathrm{mmHg}$ is not recommended in patients with $\mathrm{T} 2 \mathrm{DM}$, including older adults. However, many patients still remain below the recommended $\mathrm{BP}$ range, and the picture gets worse in patients of advanced age. Various cohort studies have shown that age-adjusted prevalence of hypertension has remained stable in older people since late 1990s but that there has been a downtrend of mean SBP and DBP values and an improvement in BP control rates [39-41]. However, the numbers of patients aged $>80$ years with seriously low BP, users of $\geq 3$ antihypertensives, and frail elderly individuals receiving excessive antihypertensive treatment have also significantly increased [39-41]. The proportion of patients with T2DM overtreated for BP was relatively lower in the present study compared to previous studies, 
possibly be due to the selection of at least two antihypertensives as a criterion of hypertension. Interestingly, whereas the participants with overtreatment for glycemia were in a healthier condition, participants with overtreatment for BP had a worse disease status, suggesting that any relation between a lower BP and improved diabetes management was unlikely. Moreover, there was only a single independent predictor of BP overtreatment which was the use of an insulin-based treatment. Even though triple combinations of antihypertensive drugs may not increase the risk of adverse events compared to dual combinations in the general population, adverse events, such as dizziness, peripheral edema, and headache, are more common among the former [42], which may be more troublesome in an older adult with T2DM.

In the presence of unnecessarily low HbA1c values, physicians in our study deintensified antihyperglycemic treatment in only a small percentage of patients. Not surprisingly, reports from other health systems are not much different, and around two-thirds of overtreated individuals continue with their current antihyperglycemic regimen [22, 40]. In previous survery, the numbers are also similar regarding the withdrawal or dose reduction of antihypertensives in subjects with excessively low BP $[22,40,43]$. In our study, physician reaction to uncontrolled glycemia differed greatly from that to uncontrolled BP. While glucose-lowering therapy was intensified in most patients with uncontrolled glycemia, $<10 \%$ of those with uncontrolled BP had their treatment modified. The decision to withdraw a medication is influenced by multiple barriers and enablers from both the patients and physicians. Skepticism on the appropriateness of withdrawing, absence of a process for cessation, and dislike of medications are the potential barriers and enablers of the patients [44]. On the physician side, problem awareness, inertia secondary to lower perceived value for stopping or continuing, self-efficacy or ability to alter prescribing, and feasibility of altering prescribing in routine care environments were identified as barriers or enablers [45]. In addition, systemlevel barriers (e.g., policy, finance) and environmental factors [46] may also delay the time of deprescribing. However, with the available data from ourstudy, we are unable to comment on the actual causes of physicians' inertia to modify antihypertensive and antiglycemic medications. In order to effectively facilitate deprescribing in clinical practice, future studies are needed to better identify barriers and enablers specific to patients with diabetes mellitus and their physicians.

Our study has a number of limitations. First, since the TEMD study was conducted in a population aged $\geq 18$ years, some variables that are more specific to older adults were not available, such as fall assessment, cognitive functions, functional status, and symptoms of depression. Second, the study sites were tertiary care centers, which makes it challenging to translate the findings to general services. Third, as there is no consensus on the definitions for diabetes overtreatment and hypertension overtreatment, we applied a modified set of criteria from existing studies. Therefore, the number of affected individuals may be different from those in other studies. Moreover, we do not have any data showing that patients had suffered from the harmful effects of overtreatment. Finally, we were not able to identify potential factors to influence deintensification or intensification of medications.

\section{CONCLUSIONS}

In conclusion, the findings of the present study indicate that overtreatment and undertreatment of glycemia and BP are common among Turkish older adults with T2DM. Deintensification of treatment in overtreated subjects is not routine among tertiary care center physicians, who also seemed more confident with the decision to intensify glucose-lowering treatment but not BP-lowering treatment. Our findings point to a need to improve physician awareness of drug modification in older patients with T2DM. 


\section{ACKNOWLEDGEMENTS}

The authors acknowledge all of the physicians and nurses at each of the TEMD study centers participating in patient recruitment and characterization (see ESM).

Funding. The TEMD study was funded by the Turkish Society of Endocrinology and Metabolism (TEMD) (Grant no. 04/10/2016-01). Automated blood pressure monitors were provided by Sanovel A/S. The funding agencies had no role in the design and conduct of the study; the collection, management, analysis, and interpretation of the data; or the preparation, review, or approval of the manuscript. No Rapid Service Fee was received by the journal for the publication of this article.

Authorship. All named authors meet the International Committee of Medical Journal Editors (ICMJE) criteria for authorship for this article, take responsibility for the integrity of the work as a whole, and have given their approval for this version to be published.

Authorship Contributions. Alper Sonmez and Ilker Tasci share the first authorship.

Prior Presentation. Part of this study was presented at the American Diabetes Association Scientific Sessions, Orlando, FL, USA, 22-26 June 2018.

Disclosures. Alper Sonmez, Ilker Tasci, Ibrahim Demirci, Cem Haymana, Cem Barcin, Hasan Aydin, Sevki Cetinkalp, Feyza Yener Ozturk, Kamile Gul, Tevfik Sabuncu, Ilhan Satman and Fahri Bayram have nothing to disclose.

Compliance with Ethics Guidelines. The study was conducted in accordance with the Helsinki Declaration of 1964, as revised in 2013. The local (University of Health Sciences, Kecioren Training and Research Hospital Ethical Committee: no. 131309) and central (Republic of Turkey, Ministry of Health, Pharmaceuticals and Medical Devices Agency: no. 14-MAR-2017/ 93189304-514.11.01-E.58933) ethics committees approved the study protocol, and participants provided informed consent to participate. This study is registered at ClinicalTrials.gov (NCT 03455101).

Data Availability. The datasets during and/ or analyzed during the current study are available from the corresponding author on reasonable request.

Open Access. This article is licensed under a Creative Commons Attribution-NonCommercial 4.0 International License, which permits any non-commercial use, sharing, adaptation, distribution and reproduction in any medium or format, as long as you give appropriate credit to the original author(s) and the source, provide a link to the Creative Commons licence, and indicate if changes were made. The images or other third party material in this article are included in the article's Creative Commons licence, unless indicated otherwise in a credit line to the material. If material is not included in the article's Creative Commons licence and your intended use is not permitted by statutory regulation or exceeds the permitted use, you will need to obtain permission directly from the copyright holder. To view a copy of this licence, visit http://creativecommons.org/licenses/bync/4.0/.

\section{REFERENCES}

1. Hogan P, Dall T, Nikolov P, et al. Economic costs of diabetes in the US in 2002. Diabetes Care. 2003;26: 917-32.

2. Gagliardino JJ, Atanasov PK, Chan JCN, et al. Resource use associated with type 2 diabetes in Africa, the Middle East, South Asia, Eurasia and Turkey: results from the International Diabetes Management Practice Study (IDMPS). BMJ Open Diabetes Res Care. 2017;5:e000297.

3. Ogurtsova K, da Rocha Fernandes JD, Huang Y, et al. IDF Diabetes Atlas: Global estimates for the prevalence of diabetes for 2015 and 2040. Diabetes Res Clin Pract. 2017;128:40-50.

4. Selvin E, Coresh J, Brancati FL. The burden and treatment of diabetes in elderly individuals in the US. Diabetes Care. 2006;29:2415-9. 
5. Greenfield S, Billimek J, Pellegrini F, et al. Comorbidity affects the relationship between glycemic control and cardiovascular outcomes in diabetes: a cohort study. Ann Intern Med. 2009;151:854-60.

6. ACCORD Study Group, Cushman WC, Evans GW, et al. Effects of intensive blood-pressure control in type 2 diabetes mellitus. NEJM. 2010;362:1575-85.

7. Action to Control Cardiovascular Risk in Diabetes Study Group, Gerstein HC, Miller ME, et al. Effects of intensive glucose lowering in type 2 diabetes. New Eng J Med. 2008;358:2545-59.

8. ADVANCE Collaborative Group, Patel A, MacMahon $\mathrm{S}$, et al. Intensive blood glucose control and vascular outcomes in patients with type 2 diabetes. New Eng J Med. 2008;358:2560-72.

9. Duckworth W, Abraira C, Moritz T, et al. Glucose control and vascular complications in veterans with type 2 diabetes. NEJM. 2009;360:129-39.

10. American Diabetes Association. Older adults: standards of medical care in diabetes-2019. Diabetes Care. 2019;42:S139.

11. Davies MJ, D'Alessio DA, Fradkin J, et al. A consensus report by the American Diabetes Association (ADA) and the European Association for the Study of Diabetes (EASD). Diabetologia. 2018;2018(61): 2461-98.

12. The Society of Endocrinology and Metabolism of Turkey (SEMT) Diabetes Mellitus Working Group. Clinical practice guideline for diagnosis, treatment and follow-up of diabetes mellitus and its complications-2019. BAYT Bilimsel Araştırmalar, Ankara.

13. American Geriatrics Society Expert Panel on the Care of Older Adults with Diabetes Mellitus. Guidelines abstracted from the American Geriatrics Society Guidelines for improving the care of older adults with diabetes mellitus: 2013 update. J Am Geriatr Soc. 2013;2013(61):2020-6.

14. American Diabetes Association. 2. Classification and diagnosis of diabetes: standards of medical care in diabetes-2018. Diabetes Care. 2018;41:S13-27.

15. AGS Choosing Wisely Workgroup. American Geriatrics Society identifies five things that healthcare providers and patients should question. J Am Geriatr Soc. 2013;61:622-31.

16. de Boer IH, Bangalore S, Benetos A, et al. Diabetes and hypertension: a position statement by the American Diabetes Association. Dia Care. 2017;40: 1273-84.
17. Aydoğdu S, Güler K, Bayram F, et al. 2019 Turkish hypertension consensus report. Turk Kardiyol Dern Ars. 2019;47:535-46.

18. Tasci I, Safer U, Naharci I, Sonmez A. Mismatch between ADA and AGS recommendations for glycated hemoglobin targets for older adults. Prim Care Diabetes. 2018;12:192-4.

19. Sonmez A, Haymana C, Bayram F, et al. Turkish Nationwide Survey of glycemic and other metabolic parameters of patients with diabetes mellitus (TEMD study). Diabetes Res Clin Pract. 2018;146:138-47.

20. Friedewald WT, Levy RI, Fredrickson DS. Estimation of the concentration of low-density lipoprotein cholesterol in plasma, without use of the preparative ultracentrifuge. Clin Chem. 1972;18:499-502.

21. Maciejewski ML, Mi X, Sussman J, et al. Overtreatment and deintensification of diabetic therapy among medicare beneficiaries. J Gen Internal Med. 2018;33:34-41.

22. Sussman JB, Kerr EA, Saini SD, et al. Rates of deintensification of blood pressure and glycemic medication treatment based on levels of control and life expectancy in older patients with diabetes mellitus. JAMA Intern Med. 2015;175:1942-7.

23. Godino JG, Appel LJ, Gross AL, et al. Diabetes, hyperglycemia, and the burden of functional disability among older adults in a community-based study. J Diabetes. 2017;9:76-84.

24. Fried TR, Niehoff K, Tjia J, Redeker N, Goldstein MK. A Delphi process to address medication appropriateness for older persons with multiple chronic conditions. BMC Geriatr. 2016;16:67-8.

25. Blackburn DF, Swidrovich J, Lemstra M. Non-adherence in type 2 diabetes: practical considerations for interpreting the literature. Patient Prefer Adher. 2013;7:183-9.

26. Egede LE, Gebregziabher $\mathrm{M}$, Dismuke $\mathrm{CE}$, et al. Medication nonadherence in diabetes: longitudinal effects on costs and potential cost savings from improvement. Diabetes Care. 2012;35:2533-9.

27. García-Pérez L-E, Alvarez M, Dilla T, Gil-Guillén V, Orozco-Beltrán D. Adherence to therapies in patients with type 2 diabetes. Diabetes Ther. 2013;4:175-94.

28. Altuntas S, Dereli T, Yilmaz MK. Multi-criteria decision making methods based weighted SERVQUAL scales to measure perceived service quality in hospitals: a case study from Turkey. Total Qual Manag Business Excell. 2012;23:1379-95. 
29. Kerse N, Buetow S, Mainous AG, Young G, Coster G, Arroll B. Physician-patient relationship and medication compliance: a primary care investigation. Ann Fam Med. 2004;2:455-61.

30. Nicklett EJ, Liang J. Diabetes-related support, regimen adherence, and health decline among older adults. J Gerontol B Psychol Sci Soc Sci. 2010;65B: 390-9.

31. Harris R, Linn MW. Health beliefs, compliance, and control of diabetes mellitus. South Med J. 1985;78: 162-6.

32. Munshi MN, Segal AR, Suhl E, et al. Assessment of barriers to improve diabetes management in older adults: a randomized controlled study. Diabetes Care. 2013;36:543-9.

33. Lipska KJ, Ross JS, Miao Y, Shah ND, Lee SJ, Steinman MA. Potential overtreatment of diabetes mellitus in older adults with tight glycemic control. JAMA Intern Med. 2015;175:356-62.

34. Tseng C-L, Soroka O, Maney M, Aron DC, Pogach LM. Assessing potential glycemic overtreatment in persons at hypoglycemic risk. JAMA Intern Med. 2014;174:259-68.

35. Overbeek JA, Heintjes EM, Prieto-Alhambra D, et al. Type 2 diabetes mellitus treatment patterns across Europe: a population-based multi-database study. Clin Ther. 2017;39:759-70.

36. American Diabetes Association. 9 Pharmacologic approaches to glycemic treatment: standards of medical care in diabetes-2019. Diabetes Care. 2019;42:S90-102.

37. Myers MG, Reeves RA, Oh PI, Joyner CD. Overtreatment of hypertension in the community? Am J Hypertens. 1996;9:419-25.

38. Messerli FH, Mancia G, Conti CR, et al. Dogma disputed: can aggressively lowering blood pressure in hypertensive patients with coronary artery disease be dangerous? Ann Intern Med. 2006;144: 884-93.

39. Guo F, He D, Zhang W, Walton RG. Trends in prevalence, awareness, management, and control of hypertension among United States adults, 1999 to 2010. J Am Coll Cardiol. 2012;60:599-606.

40. McAlister FA, Lethebe BC, Lambe C, Williamson T, Lowerison M. Control of glycemia and blood pressure in British adults with diabetes mellitus and subsequent therapy choices: a comparison across health states. Cardiovasc Diabetol. BioMed Cent. 2018;17:27-8.

41. Bromfield SG, Bowling CB, Tanner RM, et al. Trends in hypertension prevalence, awareness, treatment, and control among US adults 80 years and older, 1988-2010. J Clin Hypertens (Greenwich). 2014;16: $270-6$.

42. Düsing R, Waeber B, Destro M, Santos Maia C, Brunel P. Triple-combination therapy in the treatment of hypertension: a review of the evidence. J Hum Hypertens. 2017;31:501-10.

43. McCoy R, Lipska K. Physicians frequently fail to deintensify treatment in older patients with diabetes and very low haemoglobin A1c or blood pressure. Evidence Based Med. 2016;21:158.

44. Reeve E, To J, Hendrix I, Shakib S, Roberts MS, Wiese MD. Patient barriers to and enablers of deprescribing: a systematic review. Drugs Aging. 2013;30:793-807.

45. Anderson K, Stowasser D, Freeman C, Scott I. Prescriber barriers and enablers to minimising potentially inappropriate medications in adults: a systematic review and thematic synthesis. BMJ Open. 2014;4:e006544.

46. Linsky A, Zimmerman KM. Provider and systemlevel barriers to deprescribing: interconnected problems and solutions. Public Policy Aging Rep. 2018;28:129-33. 